\title{
Use of neuropathic pain questionnaires in predicting persistent postoperative neuropathic pain following lumbar discectomy for radiculopathy
}

\author{
Mohammed F. Shamji, MD, PhD, FRCSC,1,2 and Alina Shcharinsky, RN(EC), MN1 \\ 'Division of Neurosurgery, Toronto Western Hospital; and 2Department of Surgery, University of Toronto, Toronto, \\ Ontario, Canada
}

OBJECTIVE Failed-back surgery syndrome has been historically used to describe extremity neuropathic pain in lumbar disease despite structurally corrective spinal surgery. It is unclear whether specific preoperative pain characteristics can help determine which patients may be susceptible to such postoperative disabling symptoms.

METHODS This prospective study analyzed surgical microdiscectomy patients treated for lumbar, degenerative, painful radiculopathy. Clinical parameters included general demographics, preoperative and postoperative clinical examination status, self-reported pain and disability scores, and neuropathic pain scores. The screening tests for neuropathic pain were the Douleur Neuropathique 4 and Leeds Assessment of Neuropathic Symptoms and Signs, with correlation tested for ordinal score and screen positivity. Multiple logistic regression analysis was used to define predictors of postoperative symptomatology.

RESULTS Twelve percent of the 250 patients with radiculopathy who underwent microdiscectomy experienced persistent postoperative neuropathic pain (PPNP) with only modest, if any, relief of leg pain. The condition was highly associated with abnormal preoperative screen results for neuropathic pain, but not sex, smoking status, or preoperative pain severity $(\alpha=0.05)$. Good correlation was seen between the 2 screening tests used in this study for both absolute ordinal score (Spearman $\rho=0.84 ; p<0.001$ ) and the threshold for terming the patient as having neuropathic pain features (Spearman $\rho=0.48 ; p<0.001$ ). Younger age at treatment also correlated with a higher likelihood of developing PPNP ( $p$ $=0.03$ ).

CONCLUSIONS This population exhibited a low overall frequency of PPNP. Higher neuropathic pain screening scores correlated strongly with likelihood of significant postoperative leg pain. Further work is required to develop more accurate prognostication tools for radiculopathy patients undergoing structural spinal surgery.

http://thejns.org/doi/abs/10.3171/2015.4.SPINE141310

KEY WORDS failed-back surgery syndrome; neuropathic pain; lumbar radiculopathy; microdiscectomy

I NTERVERTEBRAL disc herniation can cause symptomatic lumbar radiculopathy by both mechanical distortion and biochemical irritation of apposed neural elements. Surgery may be indicated in cases in which pain remains refractory to conservative management or when significant or progressive motor deficit exists; symptomatic relief is the result in a significant number of patients but not in all. ${ }^{28,29}$ Neuropathic pain is a complex pain phenotype, representing a maladaptive process of normal sensory signaling altered so that pain is felt in the absence of a nociceptive stimulus, or responses to normally noxious stimuli are enhanced. Failed-back surgery syndrome (FBSS) represents a clinical entity in which components of a patient's extremity pain persist despite structurally corrective spinal surgery that achieved the objectives of neural element decompression and spinal column stabilization, where indicated. This implies a nerve derangement beyond simple compression or inflammation, in which clinical resolution does not follow nerve root decompression. Presurgical prediction of persistent postoperative neuropathic pain (PPNP) has not been well established.

After exclusion of occult structural or stability etiologies,

ABBREVIATIONS BMI = body mass index; DN4 = Douleur Neuropathique 4; FBSS = failed-back surgery syndrome; LANSS = Leeds Assessment of Neuropathic Symptoms and Signs; ODI = Oswestry Disability Index; PPNP = persistent postoperative neuropathic pain; VAS = visual analog scale.

SUBMITTED December 24, 2014. ACCEPTED April 22, 2015.

INCLUDE WHEN CITING Published online October 9, 2015; DOI: 10.3171/2015.4.SPINE141310. 
the management of PPNP has involved a multi-tiered approach involving pharmacological, interventional, and surgical maneuvers. ${ }^{8,12}$ To assist with the formal assessment of patients with neuropathic pain, a number of screening tools have been developed to render the diagnosis more objective. The Douleur Neuropathique 4 (DN4) and Leeds Assessment of Neuropathic Symptoms and Signs (LANSS) are 2 metrics that have been applied in cases of PPNP to define the next appropriate treatment steps and monitor for improvement. Both tools have excellent sensitivity and specificity and have been investigated for application in other languages, as well as in both complex pain and community settings. ${ }^{24,26,27}$

What remains unclear is how to predict which patients are more or less likely to derive benefit from surgical intervention and whether specific pain characteristics are associated with different likelihoods of desirable outcome. Such information would help surgeons and patients achieve informed decision making when selecting an appropriate management strategy. This study involved application of the DN4 and LANSS scores preoperatively in a cohort of patients with lumbar radiculopathy.

\section{Methods}

This study was based on analysis of prospectively collected data for consecutive patients treated by microdiscectomy for lumbar radiculopathy over a 2-year period of time at a single tertiary-care neurosurgical center. Inclusion criteria included age greater than 18 years and symptomatic lumbar radiculopathy with concordant spinal MRI. Exclusion criteria included a history of spinal surgery, comorbid peripheral neuropathy or peripheral vascular disease, or symptomatic cervical spondylotic disease. All procedures were performed via a posterior surgical approach with the patient in the prone position and under general anesthesia. Ethics approval from the Research Ethics Board at the Toronto Western Hospital was acquired prior to engaging in this study, with all patients consenting to data acquisition.

Demographic data collected included sex, age, smoking status, body mass index (BMI), and key comorbidities. Disease-specific clinical data collected measures of lumbar spinal disease severity, including visual analog scale (VAS) back and leg pain scores and the Oswestry Disability Index (ODI) scores, acquired both preoperatively and postoperatively. Neuropathic pain scores were acquired using the DN4 and LANSS neuropathic pain tools.

A diagnosis of PPNP was rendered if the patient underwent surgery and had persistent extremity pain with neuropathic features still present 6 months postoperatively with no identified etiology. If the extremity pain had not resolved by 3 months, patients underwent an array of tests, including Gd-enhanced MRI and dynamic radiography to rule out persistent compression and dynamic instability. If recurrent herniation or iatrogenic instability or deformity was detected, this was corrected in a second surgical procedure.

\section{Statistical Analysis}

The study population is described by descriptive statistics of demographic data and preoperative general pain, disability, and neuropathic pain scores. Spearman correlation coefficients were used to define the relationship between DN4 and LANSS scores. Principal components and factor analysis using scree plots to define the number of factors and the varimax rotation method for performing the analysis were used to determine whether each of the LANSS and DN4 comprised independent domains of neuropathic pain in the radiculopathy population. Pain, disability, and neuropathic pain scores were compared between PPNP and non-PPNP groups by the Wilcoxon rank-sum test. Sensitivity and specificity of the DN4 and LANSS for predicting PPNP status were computed. Nominal and ordinal logistic regression analyses were performed to define the dependency of postoperative clinical improvement of lumbar radiculopathy on neuropathic pain diagnosis severity. The 0.05 level of significance was used for all analyses.

\section{Results}

Analysis was performed of 250 patients with lumbar radiculopathy who had a minimum of 6 months of clinical follow-up. The average age $( \pm \mathrm{SD})$ of the patients was 57 \pm 16 years, $56 \%$ were male, and the average BMI was 28 $\pm 5 \mathrm{~kg} / \mathrm{m}^{2}$. Demographic data are summarized in Table 1 .

Preoperative general pain and disability scores are summarized in Table 2 . The mean $( \pm$ SD) VAS leg pain score was $8.2 \pm 1.2$, a result that was no different between the PPNP and non-PPNP patients. The improvement in the overall cohort was dichotomous, with the majority of patients (88\%) exhibiting substantial reduction in leg pain severity, whereas the VAS scores of $12 \%$ of patients failed to improve by more than $50 \%$. These groups differed by both the change in VAS score $(p<0.001)$ and its final absolute value $(p<0.001)$. In the group that achieved meaningful improvement, the final VAS score was $1.6 \pm$ 1.0 , whereas that of the PPNP group changed minimally to $7.0 \pm 1.2$ (p <0.001) (Fig. 1). As expected, those patients who exhibited PPNP had less improvement in pain-related disability scores (ODI, $p<0.001)$. The mean $( \pm$ SD) VAS score for back pain was $6.3 \pm 1.4$ for the entire cohort, and scores were equivalent in both groups. Improvement in the overall cohort was, again, dichotomous, with both groups demonstrating postoperative improvement in VAS back pain score, although to a lesser extent for the PPNP population $(p=0.04)$. These changes and the between-group differences in pain and disability scores are summarized in Table 3. The patients who did not benefit from surgery were generally younger than those with clinical resolution of symptoms $(\mathrm{p}=0.03)$, although sex and smoking status were not significantly different between groups.

Neuropathic pain scores are detailed and summarized in Table 2. In the overall cohort, the median DN4 score was 3 (range 1-8), with $22 \%$ meeting the threshold criteria of pain with neuropathic features. The median LANSS score was 7 (range 2-19), with 11\% meeting the threshold criteria for pain with neuropathic features. There were no specific associations between age (DN4, $\mathrm{p}=0.73$; LANSS, $\mathrm{p}=0.33)$, sex (DN4, $\mathrm{p}=0.21$; LANSS, $\mathrm{p}=0.30)$, preoperative VAS leg pain score (DN4, $p=0.29$; LANSS, $p=0.24$ ), and positive DN4 or LANSS screen results for neuropath- 
TABLE 1. Demographic characteristics of lumbar radiculopathy patients*

\begin{tabular}{lcccc}
\hline Characteristic & Entire Cohort & Non-PPNP Patients & PPNP Patients & $p$ Value \\
\hline Mean age, yrs \pm SD & $58 \pm 16$ & $59 \pm 16$ & $51 \pm 18$ & 0.03 \\
\hline Male patients & $56 \%$ & $57 \%$ & $48 \%$ & 0.38 \\
\hline Smoker & $28 \%$ & $27 \%$ & $35 \%$ & 0.56 \\
\hline Mean BMl & $28 \pm 7$ & $26 \pm 9$ & $29 \pm 12$ & 0.42 \\
\hline
\end{tabular}

* Mean values are presented \pm SD.

ic pain. There was significant correlation between the absolute neuropathic pain scores (Spearman $\rho=0.84$; $p<$ 0.001 ) and the threshold for terming the patient as having neuropathic pain features (Spearman $\rho=0.48$; $p<0.001$ ).

The factor analysis for the DN4 revealed a 5 -factor solution that explained $78 \%$ of the cumulative variance. The factor analysis for the LANSS revealed a 4-factor solution that explained $81 \%$ of the cumulative variance. Review of the score contributions to these factors reveals that both scores have discrete subcomponents of different pain characterizations, including paresthesias, altered sensation, burning pain and allodynia, shooting radicular pain, and a mixed category for the DN4 of unusual symptom patterns that occur rarely. These are summarized in Table 4. Post hoc significance testing revealed that no additional factors were necessary to further augment the analyses.

When comparing the patients achieving clinical resolution with those in the PPNP group, preoperative VAS leg pain scores were no different. Conversely, both the DN4 and LANSS scores were higher in the PPNP group (DN4 and LANSS, p < 0.001) (Fig. 2) with more frequent determination preoperatively of the patients as having neuropathic features (DN4 and LANSS, p < 0.001) (Fig. 3). Furthermore, incrementally higher preoperative neuropathic pain scores for both systems were associated with progressively higher likelihood of the patient exhibiting persistence of extremity complaints. This is shown in Fig. 4 for the DN4, where, toward the higher end of scoring system, nearly all patients exhibited postoperative pain. These comparisons are summarized in Table 2, and the computed sensitivity and specificity of the neuropathic pain scores for FBSS were 78\% and 85\% for the DN4 and $71 \%$ and $96 \%$ for the LANSS, respectively. The positive and negative predictive values for FBSS were $40 \%$ and 97\% for the DN4 and $70 \%$ and $96 \%$ for the LANSS, respectively.
Results of the nominal and ordinal logistic regression models for FBSS, including age, sex, smoking status, preoperative VAS leg pain score, and screen positivity for neuropathic pain revealed that identification of neuropathic pain with either the DN4 $(p=0.005)$ or LANSS $(p=$ 0.001) was associated with a strong likelihood of PPNP. The same held true for the postoperative change in VAS leg pain score (DN4, $\mathrm{p}<0.001$; LANSS, $\mathrm{p}<0.001)$ and final VAS leg pain score (DN4, p < 0.001; LANSS, $\mathrm{p}<$ $0.001)$. The results of the logistic regression analyses for the PPNP diagnosis are shown in Table 5. The receiver operating characteristic plots for this regression analysis had area under the curve values of 0.88 and 0.87 for DN4 and LANSS, respectively.

\section{Discussion}

The incidence of FBSS is generally reported as between $10 \%$ and $40 \%$ of spine surgical patients and varies significantly across various series, in part because it represents a diverse range of patients who may have persistent compression, dynamic instability, or true PPNP., ${ }^{1,6,11,14}$ The estimate heterogeneity likely stems from variation in patient factors, preoperative evaluation and selection for surgery, intraoperative technique, and postoperative diagnostic evaluation culminating in this neuropathic pain diagnosis. A number of studies have sought to define the predictors of outcome following surgery for lumbar radiculopathy. Barrios and coworkers examined epidemiological predictors in a cohort of 150 patients, among whom 51\% and 36\% reported excellent and good outcome, respectively. ${ }^{2}$ In their analysis, patients performing heavy work and those with prominent motor or sensory deficit were less likely to achieve desirable outcomes, with no effect of age, sex, surgical level, or intraoperative surgical details.

The prospective cohort of surgical radiculopathy pa-

TABLE 2. Preoperative pain and disability scores*

\begin{tabular}{lcccc}
\hline Pain/Disability Score & Entire Cohort & Non-PPNP Patients & PPNP Patients & $p$ Value \\
\hline Mean VAS back pain & $6.3 \pm 1.4$ & $6.3 \pm 1.5$ & $6.2 \pm 1.4$ & 0.94 \\
\hline Mean VAS leg pain & $8.2 \pm 1.2$ & $8.2 \pm 1.2$ & $8.3 \pm 1.1$ & 0.52 \\
\hline Mean ODI & $51 \pm 10$ & $50 \pm 12$ & $52 \pm 15$ & 0.81 \\
\hline Mean DN4 (IQR) & $3(1-3)$ & $3(1-3)$ & $6(4-6)$ & $<0.001$ \\
\hline DN4 (neuropathic), $\%$ & 21 & 15 & 78 & $<0.001$ \\
\hline Mean LANSS (IQR) & $7(2-7)$ & $7(2-7)$ & 70 & $<0.001$ \\
\hline LANSS (neuropathic), $\%$ & 11 & 4 & & $<0.001$ \\
\hline IQR $=$ interquartile range. \\
$*$ Mean values are presented \pm SD unless otherwise noted.
\end{tabular}




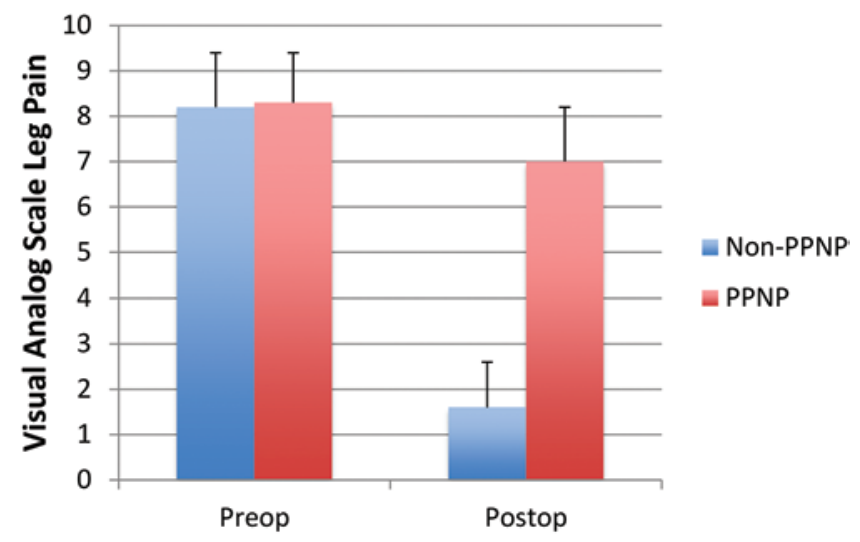

FIG. 1. Preoperative and postoperative VAS leg pain scores for patients with and without FBSS. There was no difference in preoperative VAS scores in the 2 groups $(p=0.52$ ). Figure is available in color online only.

tients in the current study exhibited an expected fraction of patients in whom a substantial improvement in leg pain failed to occur despite structurally corrective spinal surgery. This is different from the cohort of patients who exhibited recurrence of their radicular pain phenotype, which is more typical of a recurrent disc herniation that is most common among patients with comorbid obesity or smoking status..$^{19}$ This PPNP population was characterized by less postoperative improvements in VAS leg and back pain scores than in the non-PPNP population. Nevertheless, clinically significant improvement in disability was observed in both groups, suggesting practical overall benefit to the patient despite the persistence of significant pain and hypersensitivity. Screen positivity for neuropathic pain by the DN4 or the LANSS questionnaires did prognosticate the occurrence of PPNP, whereas sex, smoking status, BMI, and surgical level bore no correlation with this outcome.

Voorhies and coworkers sought to understand pain characteristics prognosticating worse outcome in a cohort of 110 patients in whom there was substantial discordance between patient self-reported outcome and surgeonreported outcome: $26 \%$ of patients and $55 \%$ of surgeons reported the outcome to be excellent. ${ }^{25}$ Nevertheless, in both groups, there were a number of preoperative predictors that excellent or good outcome would be less likely to achieve, including historical features of axial joint pain and compensation claim and screening features of highseverity Modified Ransford Score pain drawing and McGill Pain Questionnaire affective and sensory scores. The conclusions of this work are important in defining the psychosocial factors that may impact patient satisfaction following structural spinal surgery.
Indeed, workup of the patient with persistence of lower-extremity pain following structurally corrective spinal surgery involves reassessment of the original radiculopathy diagnosis and also investigation of the presence of ongoing static compression or dynamic instability. ${ }^{6}$ These considerations must include reassessment of the surgical level and side, ${ }^{20}$ adequacy of decompression, absence of occult or iatrogenic instability, and occurrence of surgical complications or recurrent disease. ${ }^{16}$

The diagnosis of FBSS has been variably applied from its inception to include any time when patient outcome is not in line with expectations of surgery to actual neuropathic pain diagnosis in the absence of static or dynamic structural disease. When applying the diagnosis specifically to cases of PPNP, it is made when further structural surgery is not indicated. The next stages of innovation to prevent its occurrence will involve preoperative optimization of patient selection to exclude this cohort from surgery, appropriate and judicious application of neuropathic pain management from pharmacological to neuromodulatory, ${ }^{12,15}$ and the development of molecular and electrophysiological therapeutics to reverse the aberrant neurophysiology in this population., ${ }^{9,10,18}$ The work presented herein will aid in the first of these objectives, by informing surgeons and patients about prognostic features that portend low likelihood of clinical benefit to be conferred by structural spinal surgery.

The DN4 and LANSS questionnaires had good correlation with screening for neuropathic pain in this cohort, and the significant fraction with a positive screen is a group that should not be ignored. The regression analysis strongly associating both tools with the PPNP outcome, with the overall change in VAS leg pain score, and with the final VAS leg pain score, suggests that there is value in screening for these features to enable better counseling of patients considering structurally corrective spinal surgery for lumbar radiculopathy. While the thresholds for diagnosing pain with neuropathic features (DN4 $\geq 4$; LANSS $\geq 12$ ) have been validated for these tools, the opposite goal of identifying radicular pain responsive to structural spinal surgery is unlikely to have the same threshold values. Consequently, the modest positive and excellent negative predictive values for these tools at predicting PPNP might be advantageously shifted if other threshold scores were used. Such sensitivity analysis is an important follow-up goal to be implemented when a larger and multicenter data set is available.

The prevalence of chronic pain among working-age adults in Canada has been reported to be $16 \%-41 \%$, is more frequent among women and with advancing age, and carries high social and economic impact. ${ }^{3,13,17,22}$ Models of health care are evolving from predominantly focusing on

TABLE 3. Postoperative pain and disability scores*

\begin{tabular}{ccccc}
\hline Pain/Disability Score & Entire Cohort & Non-PPNP Patients & PPNP Patients & $p$ Value \\
\hline Mean VAS back pain & $3.9 \pm 1.5$ & $3.7 \pm 1.6$ & $4.9 \pm 1.4$ & 0.04 \\
\hline Mean VAS leg pain & $2.2 \pm 2.0$ & $1.6 \pm 1.0$ & $7.0 \pm 1.2$ & $<0.001$ \\
\hline Mean ODI & $23 \pm 14$ & $22 \pm 10$ & $33 \pm 12$ & $<0.001$ \\
\hline * Mean values are presented \pm SD. & & &
\end{tabular}


TABLE 4. Principal components analysis of neuropathic pain scores*

\begin{tabular}{|c|c|c|c|c|c|}
\hline Pain Component & Factor 1 & Factor 2 & Factor 3 & Factor 4 & Factor 5 \\
\hline DN4 cumulative $\%$ of variance & 21.3 & 41.4 & 55.9 & 67.8 & 78.5 \\
\hline Burning & 0.08 & 0.20 & 0.81 & -0.05 & 0.18 \\
\hline Painful cold & 0.03 & 0.02 & 0.21 & 0.87 & 0.11 \\
\hline Electric shocks & -0.59 & -0.38 & -0.09 & -0.31 & 0.10 \\
\hline Tingling & 0.94 & 0.01 & 0.07 & -0.06 & 0.12 \\
\hline Pins \& needles & 0.93 & -0.10 & -0.03 & 0.03 & 0.03 \\
\hline Numbness & 0.09 & 0.50 & -0.16 & 0.50 & -0.27 \\
\hline Itching & 0.08 & 0.04 & 0.01 & 0.04 & 0.95 \\
\hline Hypoesthesia (touch) & 0.06 & 0.90 & 0.10 & 0.06 & 0.06 \\
\hline Hypoesthesia (prick) & -0.06 & 0.88 & 0.22 & 0.01 & 0.04 \\
\hline Brush allodynia & 0.00 & 0.06 & 0.80 & 0.26 & -0.16 \\
\hline LANSS cumulative $\%$ of variance & 30.8 & 50.7 & 66.4 & 81.2 & \\
\hline Tingling/pins \& needles & 0.12 & 0.88 & -0.18 & -0.11 & \\
\hline Discolored & 0.16 & 0.06 & -0.05 & 0.96 & \\
\hline Hypersensitivity & 0.93 & 0.10 & 0.04 & 0.13 & \\
\hline Electric shocks & -0.03 & -0.77 & -0.31 & -0.26 & \\
\hline Burning & 0.62 & 0.12 & 0.33 & 0.09 & \\
\hline Brush allodynia & 0.91 & 0.00 & 0.04 & 0.05 & \\
\hline Altered pinprick & 0.17 & 0.01 & 0.92 & -0.06 & \\
\hline
\end{tabular}

* Values in boldface type are significant at the 0.05 level.

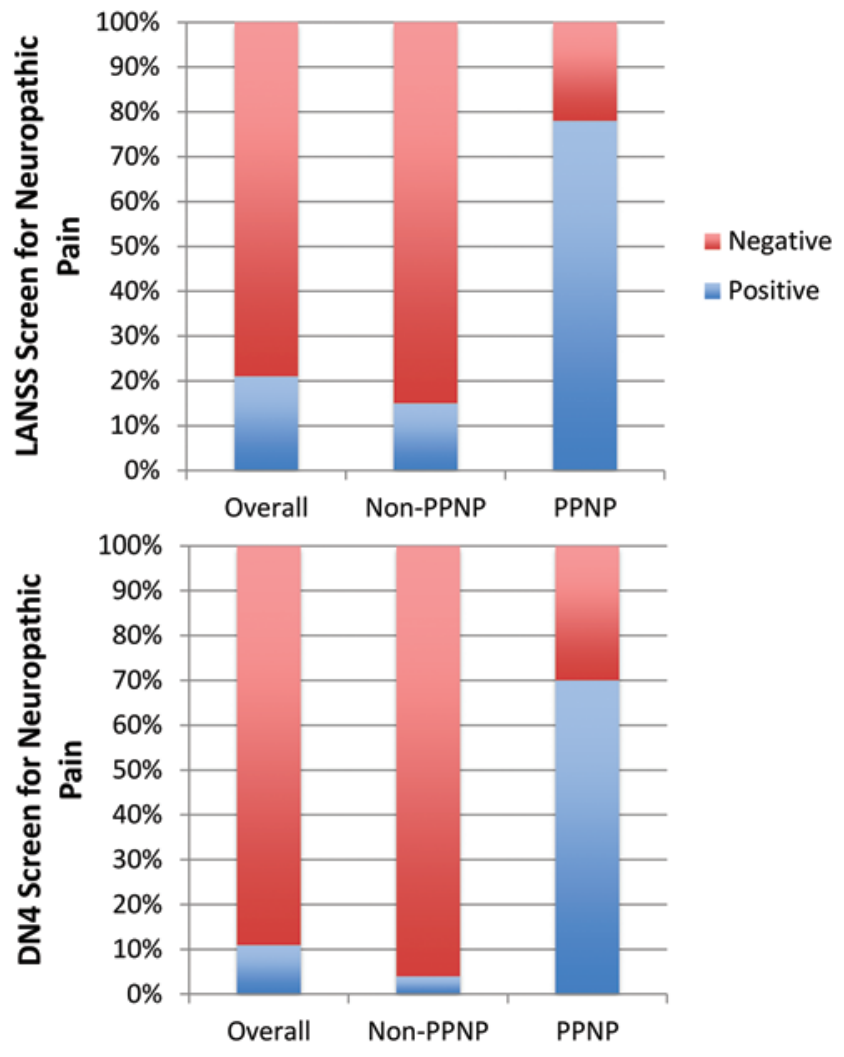

FIG. 2. Bar graphs of screening tool positivity. Upper: Positivity of LANSS neuropathic pain screen for the overall cohort of patients with lumbar radiculopathy. Lower: Positivity of DN4 neuropathic pain screen for the overall cohort. Significant preoperative differences in screen positivity were observed for patients who experienced clinical resolution compared with those with PPNP. Figure is available in color online only.
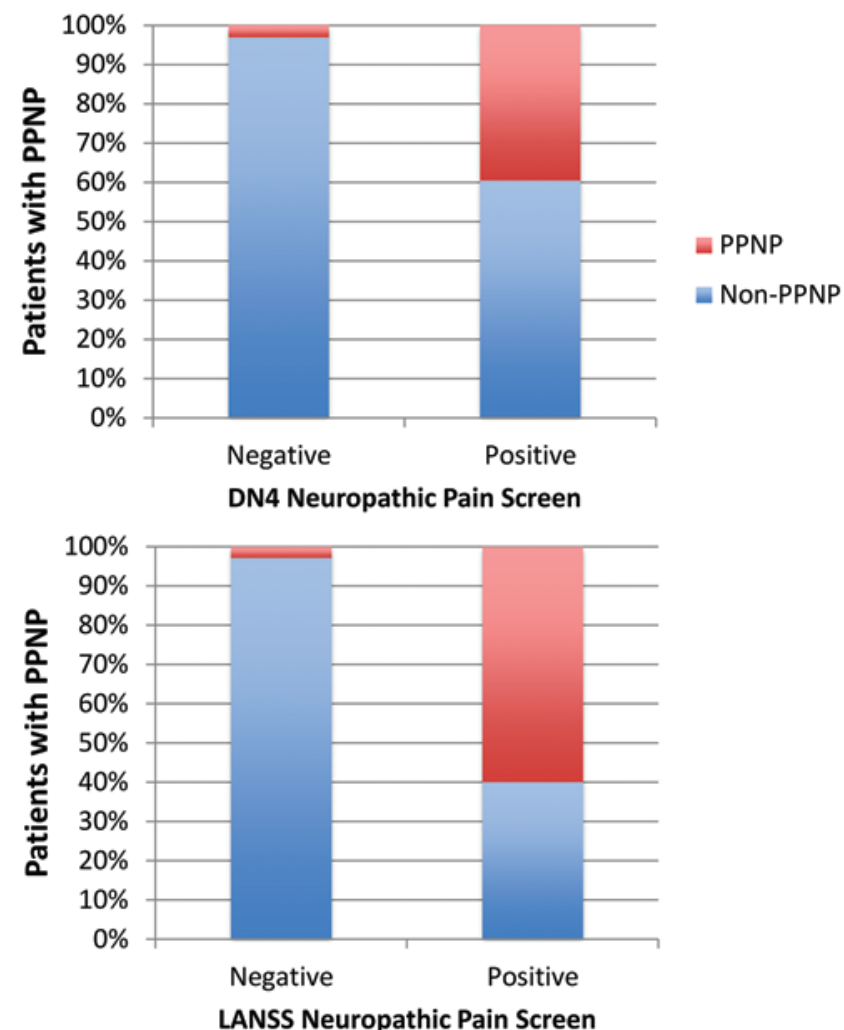

FIG. 3. Predictive value of the LANSS and DN4 neuropathic pain screening tests. Upper: DN4 positivity for PPNP development. Lower: LANSS positivity for PPNP development. Postoperative neuropathic pain was seen more frequently in those with screen positivity but not uniformly so. Figure is available in color online only. 


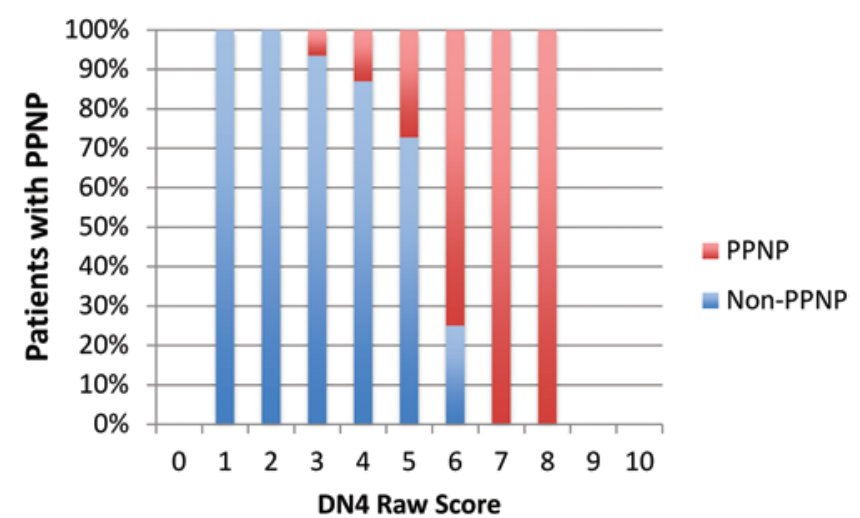

FIG. 4. Bar graph of the predictive value of absolute DN4 neuropathic pain screen scores for the development of PPNP. Postoperative neuropathic pain was seen more frequently with higher absolute screen scores but not uniformly so. Figure is available in color online only.

cure-oriented strategies to comprehensive care of chronic conditions and their substantial impact on quality of life. Poor outcomes of pain management have been reported among patients with pain of prolonged duration, pain-related catastrophizing behavior, history of abuse or trauma, or comorbid psychological distress..$^{5,7,23}$ Neuropathic features of the pain occur with less frequency, 4,17,21 although its management remains challenging and often refractory to pharmacological therapy. For such patients, the longevity of managing their pain diagnosis, the disability that it carries, and the complications of various treatments contribute to the development or aggravation of underlying psychological comorbidity that can affect their overall health status.

\section{Conclusions}

This cohort of surgical patients was evaluated using validated neuropathic pain screening tools to understand the presence of these features among lumbar radiculopathy patients. Good correlation was seen between the DN4 and LANSS screening tools, suggesting that a neuropathic pain diagnosis does exist among a surgical cohort of lumbar radiculopathy patients, with further findings indicating that exceeding established threshold values portends worse prognosis for postoperative recovery. These findings will better inform patients and surgeons about surgical expectations and decision making for cases in which neuropathic pain features exist. Screening for such diagnosis is recommended in a complete evaluation of the spine surgical patient.

\section{References}

1. Asch HL, Lewis PJ, Moreland DB, Egnatchik JG, Yu YJ, Clabeaux DE, et al: Prospective multiple outcomes study of outpatient lumbar microdiscectomy: should 75 to $80 \%$ success rates be the norm? J Neurosurg 96 (1 Suppl):34-44, 2002

2. Barrios C, Ahmed M, Arrotegui JI, Björnsson A: Clinical factors predicting outcome after surgery for herniated lumbar disc: an epidemiological multivariate analysis. J Spinal Disord 3:205-209, 1990

3. Birse TM, Lander J: Prevalence of chronic pain. Can J Public Health 89:129-131, 1998

4. Bouhassira D, Lantéri-Minet M, Attal N, Laurent B, Touboul C: Prevalence of chronic pain with neuropathic characteristics in the general population. Pain 136:380-387, 2008

5. Campbell CM, Jamison RN, Edwards RR: Psychological screening/phenotyping as predictors for spinal cord stimulation. Curr Pain Headache Rep 17:307, 2013

6. Chan CW, Peng P: Failed back surgery syndrome. Pain Med 12:577-606, 2011

7. Dunn KM, Croft PR: The importance of symptom duration in determining prognosis. Pain 121:126-132, 2006

8. Hussain A, Erdek M: Interventional pain management for failed back surgery syndrome. Pain Pract 14:64-78, 2014

9. Hwang PY, Allen KD, Shamji MF, Jing L, Mata BA, Gabr MA, et al: Changes in midbrain pain receptor expression, gait and behavioral sensitivity in a rat model of radiculopathy. Open Orthop J 6:383-391, 2012

10. Kobayashi S, Yoshizawa H, Yamada S: Pathology of lumbar nerve root compression. Part 2: morphological and immunohistochemical changes of dorsal root ganglion. J Orthop Res 22:180-188, 2004

11. Lehmann TR, LaRocca HS: Repeat lumbar surgery. A review of patients with failure from previous lumbar surgery treated by spinal canal exploration and lumbar spinal fusion. Spine (Phila Pa 1976) 6:615-619, 1981

12. Moulin DE, Clark AJ, Gilron I, Ware MA, Watson CP, Sessle $\mathrm{BJ}$, et al: Pharmacological management of chronic neuropathic pain - consensus statement and guidelines from the Canadian Pain Society. Pain Res Manag 12:13-21, 2007

13. Moulin DE, Clark AJ, Speechley M, Morley-Forster PK: Chronic pain in Canada-prevalence, treatment, impact and the role of opioid analgesia. Pain Res Manag 7:179-184, 2002

14. North RB, Campbell JN, James CS, Conover-Walker MK, Wang H, Piantadosi S, et al: Failed back surgery syndrome: 5 -year follow-up in 102 patients undergoing repeated operation. Neurosurgery 28:685-691, 1991

15. North RB, Kidd DH, Farrokhi F, Piantadosi SA: Spinal cord stimulation versus repeated lumbosacral spine surgery for chronic pain: a randomized, controlled trial. Neurosurgery 56:98-107, 2005

16. Pope MH, Goh KL, Magnusson ML: Spine ergonomics. Annu Rev Biomed Eng 4:49-68, 2002

17. Schopflocher D, Taenzer P, Jovey R: The prevalence of chronic pain in Canada. Pain Res Manag 16:445-450, 2011

TABLE 5. Multivariate logistic regression for prediction of PPNP

\begin{tabular}{|c|c|c|c|c|}
\hline \multirow[b]{2}{*}{ Component } & \multicolumn{2}{|c|}{ DN4 } & \multicolumn{2}{|c|}{ LANSS } \\
\hline & OR & $p$ Value & OR & p Value \\
\hline Age (per yr) & $1.03(1.01,1.07)$ & 0.013 & $1.04(1.00,1.07)$ & 0.047 \\
\hline $\operatorname{Sex}(F / M)$ & $1.01(0.38,2.73)$ & 0.98 & $1.01(0.33,3.22)$ & 0.99 \\
\hline Smoking (yes/no) & $1.02(0.54,2.01)$ & 0.93 & $1.03(0.47,2.98)$ & 0.97 \\
\hline Preop VAS leg pain score (per unit) & $1.03(0.67,1.57)$ & 0.89 & $1.23(0.77,2.02)$ & 0.39 \\
\hline Neuropathic pain (score threshold diagnosis) & $24(9,73)$ & $<0.001$ & $74(24,271)$ & $<0.001$ \\
\hline
\end{tabular}


18. Shamji MF, Allen KD, So S, Jing L, Adams SB Jr, Schuh R, et al: Gait abnormalities and inflammatory cytokines in an autologous nucleus pulposus model of radiculopathy. Spine (Phila Pa 1976) 34:648-654, 2009

19. Shamji MF, Bains I, Yong E, Sutherland G, Hurlbert RJ: Treatment of herniated lumbar disk by sequestrectomy or conventional diskectomy. World Neurosurg 82:879-883, 2014

20. Shamji MF, Hurlbert RJ: Oops, I did it again.... World Neurosurg 79:448-450, 2013

21. Toth C, Lander J, Wiebe S: The prevalence and impact of chronic pain with neuropathic pain symptoms in the general population. Pain Med 10:918-929, 2009

22. Tripp DA, VanDenKerkhof EG, McAlister M: Prevalence and determinants of pain and pain-related disability in urban and rural settings in southeastern Ontario. Pain Res Manag 11:225-233, 2006

23. Tunks ER, Crook J, Weir R: Epidemiology of chronic pain with psychological comorbidity: prevalence, risk, course, and prognosis. Can J Psychiatry 53:224-234, 2008

24. Unal-Cevik I, Sarioglu-Ay S, Evcik D: A comparison of the DN4 and LANSS questionnaires in the assessment of neuropathic pain: validity and reliability of the Turkish version of DN4. J Pain 11:1129-1135, 2010

25. Voorhies RM, Jiang X, Thomas N: Predicting outcome in the surgical treatment of lumbar radiculopathy using the Pain Drawing Score, McGill Short Form Pain Questionnaire, and risk factors including psychosocial issues and axial joint pain. Spine J 7:516-524, 2007

26. Walsh J, Rabey MI, Hall TM: Agreement and correlation between the self-report Leeds Assessment of Neuropathic Symptoms and Signs and Douleur Neuropathique 4 Questions neuropathic pain screening tools in subjects with low back-related leg pain. J Manipulative Physiol Ther 35:196202, 2012
27. Weingarten TN, Watson JC, Hooten WM, Wollan PC, Melton LJ III, Locketz AJ, et al: Validation of the S-LANSS in the community setting. Pain 132:189-194, 2007

28. Weinstein JN, Lurie JD, Tosteson TD, Skinner JS, Hanscom $\mathrm{B}$, Tosteson AN, et al: Surgical vs nonoperative treatment for lumbar disk herniation: the Spine Patient Outcomes Research Trial (SPORT) observational cohort. JAMA 296:2451-2459, 2006

29. Weinstein JN, Tosteson TD, Lurie JD, Tosteson AN, Hanscom B, Skinner JS, et al: Surgical vs nonoperative treatment for lumbar disk herniation: the Spine Patient Outcomes Research Trial (SPORT): a randomized trial. JAMA 296:24412450, 2006

\section{Disclosure}

The authors report no conflict of interest concerning the materials or methods used in this study or the findings specified in this paper.

\section{Author Contributions}

Conception and design: both authors. Acquisition of data: both authors. Analysis and interpretation of data: both authors. Drafting the article: both authors. Critically revising the article: both authors. Reviewed submitted version of manuscript: both authors. Approved the final version of the manuscript on behalf of both authors: Shamji. Statistical analysis: Shamji. Administrative/technical/material support: Shamji. Study supervision: Shamji.

\section{Correspondence}

Mohammed F. Shamji, Division of Neurosurgery, Toronto Western Hospital, WW4-446, 399 Bathurst St., Toronto, ON M5T 2S8, Canada. email: mohammed.shamji@uhn.ca. 